\title{
El potencial de Tena como destino de turismo de aventura
}

\section{The potential of Tena as a destination of adventure tourism}

\author{
Denisse Espinosa ${ }^{1}$, Fernando Herrera ${ }^{1}$, Ángela Lapo ${ }^{1}$, Luis Pérez ${ }^{1}$ \\ ${ }^{1}$ Universidad Central del Ecuador. Facultad de Ciencias Agrícolas. Carrera de Turismo Ecológico. Jerónimo Leiton y Av. La Gasca s/n. Ciudadela \\ Universitaria. Quito. 170521. Ecuador
}

\begin{abstract}
Resumen
El presente artículo analiza las actividades turísticas de aventura que oferta la ciudad de Tena, Ecuador, en relación con el tipo de turista que visita el lugar, de manera particular en la provincia de Napo, en la Amazonia ecuatoriana. La ciudad de Tena ha experimentado un considerable desarrollo en infraestructura turística, además posee lugares naturales únicos en el mundo, idóneos para realizar turismo de aventura. Para determinar las características del turista de aventura en el Tena, se realizaron 100 encuestas a turistas nacionales y extranjeros. Entre los principales resultados, se obtuvo que el $83 \%$ de visitantes fueron de origen nacional y el $17 \%$, extranjero, y que en la mayor parte sus gastos se realiza en alimentación y bebida, realización de deportes de aventura y alojamiento. Se evidenció la necesidad de un plan promocional para incrementar el porcentaje de visitantes, principalmente extranjeros.
\end{abstract}

Palabras clave: Amazonia ecuatoriana, demanda turística, destino turístico, calidad de vida, Ecuador.

\begin{abstract}
The present article analyzed the adventure tourism which is offered at Tena, Ecuador and the Tourism who was coming to visit the prefecture of Napo, Ecuadorian Amazonia. The city of Tena has developed into a natural paradise and offered to tourism comfort and adventures activities which are looking for foreigner and citizens. In order to define the tourists' characteristics at Tena, it has applied a survey to 100 national and foreigner tourists. In addition, the survey shows that a major spend goes to food and beverages, adventure sports and accommodation. The result of this investigation showed of $83 \%$ of national tourism and $17 \%$ of foreigner tourism, so that, this prefecture need to have a promotional plan to increase the number of international tourism.
\end{abstract}

Keywords: Ecuadorian Amazon, tourism demand, tourism destination, quality of life, Ecuador.

\section{Introducción. El turismo de aventura}

Desde sus orígenes, el término "turismo" ha sido asociado a la acción de "viajar por placer", empleando el tiempo libre y buscando recreación. Para la Organización Mundial del Turismo (OMT), el turismo comprende actividades que realizan las personas durante sus viajes y estancias en lugares distintos al de su residencia habitual, por menos de un año y con fines de ocio, negocios, estudio, entre otros. El turismo es una actividad multisectorial muy compleja que genera, directa e indirectamente, una serie de beneficios a los distintos niveles de la sociedad. Para ello, moviliza a diversos agentes y grupos sociales de manera ordenada y planificada (MINCETUR, 2015).

Con el objeto de aclarar conceptos sobre el turismo de aventura, se analizarán a continuación las tendencias que rigen el desarrollo de estos destinos turísticos internacionales. El turismo de aventura es una modalidad de turismo, en la que, como afirma Mediano (2004, p.11.), "el turista participa de manera activa en el conocimiento del territorio visitado"; y se diferencia del turismo ecológico 
porque en este caso "la naturaleza es el elemento pasivo, siendo descubierta por el turista, quién suele realizar también actividades deportivas y a veces arriesgadas". En otro punto de vista, según la OMT (1998, p.85), el turismo de aventura corresponde a "actividades turísticas que introducen un elemento de dificultad física y en ocasiones entrañan verdadero riesgo". Entre las actividades aludidas se encuentran el descenso de cursos de agua en balsas, el andinismo, la pesca deportiva, las caminatas en la naturaleza, etc. Por otra parte, la Asociación Mundial de Turismo de Aventura promueve la siguiente definición: Viajes de "aventura" incluyen dos de los siguientes tres aspectos: actividad física, interacción con la naturaleza, e intercambio o aprendizaje cultural. Finalmente, Sung et al. (1996) precisan que el crecimiento del turismo de aventura viene acompañado de una gran variedad y disponibilidad de productos, y de viajes de aventura de carácter internacional.
En la actualidad, el turismo de aventura se ha convertido en uno de los segmentos de mercado de más rápido crecimiento, especialmente porque la variedad y disponibilidad de los productos de turismo de aventura incluye una amplia gama de intereses y habilidades, que pareciera no tener límites. Según cifras mundiales, el turismo de aventura genera un rubro de $263 \mathrm{mil}$ millones de USD, y se estima que un turista promedio gasta alrededor de 3,100 USD, sin considerar los boletos de avión para pernoctar 8.8 días (MINTUR, 2014).

En este marco, se ha considerado oportuno establecer las principales actividades que se desarrollan dentro del turismo de aventura, recogidas en la Tabla 1. Más aún, cuando al igual que cualquier demanda turística existen diferentes segmentos de mercado con variados intereses gustos y preferencias, por lo que el turismo activo busca también dar respuesta a las diferentes inquietudes del turista surgiendo una amplia oferta de actividades adaptadas a ello.

Tabla 1. Clasificación de las actividades del turismo activo/aventura.

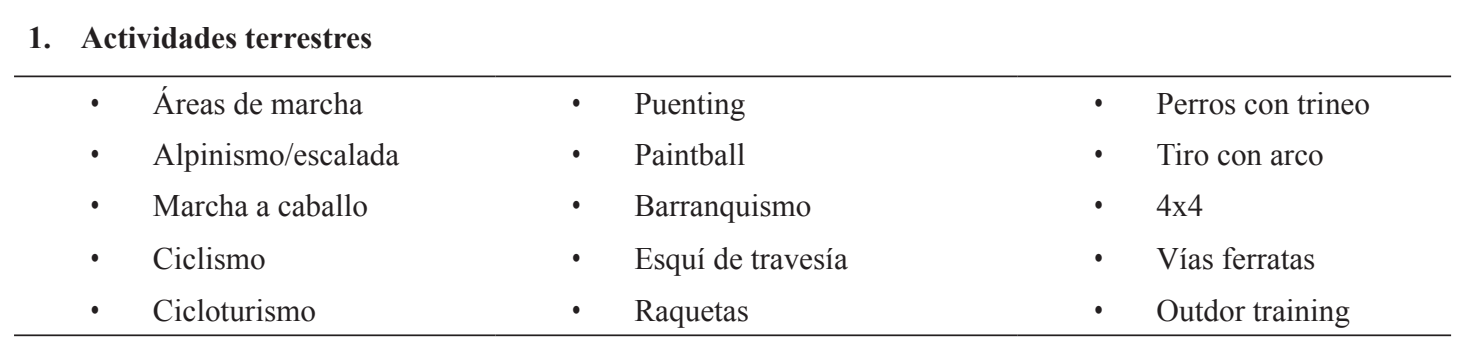

2. Actividades acuáticas

\begin{tabular}{|c|c|c|c|c|}
\hline - & Praguismo/kayak & - $\quad$ Surf/Windsurf/kitesurf & - & Hidrobob/Hidrospeed \\
\hline - & turismo fluvial & - $\quad$ Moto náutica & & Vela \\
\hline - & Buceo/submarinos & Rafting & - & Esquí acuático \\
\hline
\end{tabular}

3. Actividades aéreas

\begin{tabular}{lllll}
\hline - Ala delta & $\cdot$ & Paracaidismo de pendiente & $\bullet$ & Vuelo sin motor \\
• Globo aerostático & $\cdots$ & Paracaidismo & $\bullet$ & Hellexcursión \\
• Parapente & $\cdots$ & Ultraligeros & \\
\hline
\end{tabular}

Fuente: Elaboración propia a partir de Oliveira (1995) y Martínez \& Fernández (2011)

En Ecuador, en los últimos años se han realizado campañas para dar a conocer y conservar la riqueza natural existente, en este sentido, el turismo de aventura brinda al turista el contacto con la naturaleza, a través de sus distintas formas de deportes de aventura que buscan tanto extranjeros como ecuatorianos. Ecuador ofrece deportes de aventura catalogados, de acuerdo con la OMT, como vuelo en globo, vuelo en ala delta, vuelo en parapente, paracaidismo, buceo autónomo, buceo libre, descenso en ríos, kayaquing, pesca recreativa, montañismo, canotaje, escalada deportiva y en roca, ciclismo de montaña, caminata de alto impacto o trekking, rappel, cabalgata, entre otras. 
Con el propósito de precautelar y salvaguardar la integridad de los viajeros que realizan turismo de aventura en Ecuador, el Ministerio de Turismo, mediante Acuerdo Ministerial, expidió el Reglamento de Operación Turística de Aventura, en el cual se regula toda operación turística de aventura en el país. Ecuador ha dado un paso enorme en la consolidación de un gran proyecto, como es ofrecer un marco regulatorio moderno, adecuado y responsable para el turismo de aventura.

\section{Orígenes del Tena y de su actividad turística}

Los habitantes originarios de la zona son "Los Quijos", quienes por ser confundidos con una etnia que vivía en las faldas de la cordillera occidental fueron llamados Yumbos. Este es un grupo étnico casi extinto de las estribaciones occidentales de la provincia de Pichincha, quienes fueron obligados a desplazarse por efecto de la conquista española, llegando así a la provincia de Napo, de allí la relación existente entre los quichuas del Alto Napo y los Yumbos. Más tarde, los misioneros les dieron el nombre de "Alamas", y hoy se los conoce como "Quichuas", quienes convertidos en sus primeros habitantes, sobresalieron por su espíritu libertario y su concepto de independencia, y supieron aprovechar claramente la ruptura de relaciones entre los comerciantes blancos y los misioneros para obtener notorias ventajas en la conservación de su status de vida libre y nómada.

El oro, la canela, el ishpingo, las pieles y los animales exóticos, que abastecían la demanda serraniega, daban a la zona una fama de riqueza. Su gente se caracteriza por su forma de guerrear, por ser un pueblo fuertemente identificado entre sí, por su amor a la selva con sus propias técnicas de caza, pesca, manejo de la tierra, su mundo espiritual y su comportamiento social. De esta unión nació su cultura, religión, costumbres, tradiciones y su afán de conquista, alcanzando un significativo nivel cultural en la región.

Posteriormente, la región se pobló con los migrantes de la Sierra y la Costa que llegaron a la ciudad de Tena en calidad de colonos, y por los misioneros originarios de Europa y EEUU. Esta integración cultural marcó el inicio para que San Juan de los Dos Ríos de Tena se convierta en la provincia de Napo, y en el referente de la identidad amazónica. Su desarrollo actual es el vivo reflejo de todo este proceso cultural. Tena va tomando forma a co- mienzos del siglo XX con la construcción de la Plaza Central, marcando de esta manera el inicio del desarrollo de la ciudad. Hoy su desarrollo se sustenta en el compromiso de todos sus ciudadanos por mejorar su educación, ampliar la cobertura de salud y diversificar las distintas actividades productivas, procurando en todo momento cumplir con el objetivo del "Sumak Kawsay". Todo esto sin duda ha permitido para que Tena en la actualidad se haya convertido en un verdadero destino turístico (AME, 2014).

La ciudad de Tena presenta varios atractivos de interés natural dentro de su territorio, siendo la belleza escénica y paisajística amplia y variada debido a su ubicación geográfica. Su altitud promedio es de 512 m.s.n.m. (a nivel del centro urbano), y en las estribaciones de la Cordillera de los Llanganates la altura puede llegar a los 2,800 m.s.n.m. El clima es cálido húmedo por la abundante presencia de vegetación y bosque, y debido al variado rango altitudinal se tiene la presencia de distintos pisos altitudinales, los cuales conjugados con la temperatura promedio de 22 a $28^{\circ} \mathrm{C}$, y las precipitaciones anuales recibidas en la parroquia de $3,000 \mathrm{~mm}$, dan origen a la gran biodiversidad presente en sus bosques (Ron, 2009).

Entre los atractivos de mayor relevancia y jerarquización resaltan las cuencas hidrográficas de los ríos Tena, Pano, Jatun Yacu y Misahuallí, que atraviesan la ciudad, y el Parque Amazónico La Isla, ubicado en el centro de la ciudad en la confluencia (delta) de los ríos Tena y Pano. Ésta es un área de conservación de especies vegetales y animales, considerado a su vez como un Centro de Interpretación Ambiental. Este parque desarrolla actividades educativas a los pobladores con el fin de concientizar sobre la importancia de los ecosistemas. En sus 24 hectáreas de extensión, hasta el momento, se ha determinado la existencia de 135 especies, lo cual ha contribuido a su conservación debido a la importancia que posee a nivel nacional. Entre las especies animales más representativas se consideran las siguientes: colibrís, guanta, guatusa, armadillos, monos barizos y mono chichico de cola roja. Las especies vegetales simbólicas de la localidad son chonta duro, kili, guayusa, canela, ortiga y anona.

Otro atractivo es el Bosque Protector Colonso-Chalupas, zona de biodiversidad de reciente valoración debido a estudios ornitológicos y de mamíferos. En la actualidad, es considerado como uno de las áreas protegidas municipales de mayor importancia a 
nivel nacional, su extensión va desde las estribaciones orientales de la cordillera de los Llanganates hasta los páramos de Chalupas, en la provincia de Cotopaxi. El acceso por el cantón Tena es siguiendo la vía que se dirige por la parroquia rural de Muyuna hacia el poblado de Alto Tena-Shitig. De la cuenca del río Colonso se abastece de agua potable al cantón. Es un área con una alto índice de biodiversidad, entre sus joyas escondidas se encuentra la cascada de Pachimbi, que quiere decir el "agua donde hay mucho Pachin" (un pez de la Amazonía), con una altura de más de 30 metros. Esta caída de agua es uno de los encantos que ofrece este bosque, que con sus aguas cristalinas y el cantar de sus aves, es un paraíso por descubrir. Si se tiene suerte, con un poco de sigilo y bajo el acompañamiento de un guía de la zona, se puede apreciar al oso de anteojos, para ello se tiene que caminar en silencio, tener buenos ojos para ver en el follaje de la selva, y paciencia (Mora et al., 2014).

La mayoría de las especies de flora y fauna del cantón Tena son representativas de todas las provincias amazónicas, debido a que están situadas en un rango altitudinal similar, existiendo coincidencias de hábitats de las especies y en su distribución geográfica. Se debe considerar que a su vez existen características diferenciadoras en ciertos aspectos debido a que el cantón se encuentra rodeado por las estribaciones orientales de la cordillera de los Llanganates. De cierta manera, la variación de sus pisos altitudinales y zonas de vida hacen que la localidad cuente con variedades de flora y fauna de una mayor cantidad de hábitats, esto la diferencia de algunas provincias como Orellana y Sucumbíos.

Además, cabe resaltar la presencia de varias áreas naturales protegidas pertenecientes al $\mathrm{Pa}$ trimonio de Áreas Naturales del Estado (Parque Nacional Llanganates, Parque Nacional Sumaco, Napo Galeras, Bio Parque La Isla), así como también áreas protegidas privadas como la Estación Biológica Jatun Sacha, y los Bosques Protectores Municipales, lo cual constituye una verdadera zona de protección de los recursos naturales existentes en la localidad, para el aprovechamiento y desarrollo sostenible de las actividades turísticas vinculadas con los deportes de aventura.

Las actividades turísticas efectuadas en el territorio del Tena muestran una proyección hacia el desarrollo de actividades de naturaleza, como es la práctica de deportes extremos y deportes de aventura, realiza- dos principalmente en los ríos Jatunyacu, Anzu, Napo y Arajuno. A pesar de esta fuerte potencialidad para el desarrollo del turismo de aventura, el interés de la población por el desarrollo de las actividades turísticas está en decadencia, debido al deficiente desarrollo e innovación de los productos turísticos que oferta la localidad. Además, se ha visto superada por ciudades vecinas con las que compite, y por la ineficiente labor desempeñada por las instituciones públicas que manejan el turismo en Tena, CATUNA (Cámara de Turismo de Napo), Mintur- Dirección regional 2 y la Dirección de Biodiversidad y turismo del GAD-TENA. Estos son los principales problemas que afronta el territorio para alcanzar un trabajar conjunto, en pos de organizar las actividades turísticas.

\section{Metodología}

La investigación se realizó en el cantón Tena, provincia de Napo, Ecuador. El cantón tiene una superficie de 391,809 ha, que equivale al $11.4 \%$ de la provincia de Napo, y al $1.4 \%$ del territorio nacional. Está ubicado entre las coordenadas X: 788,047 Y: 9'897,100 m por el oeste y las coordenadas X: 273,903 Y: 9'898,213 m por el este. El sistema de proyección es UTM, Datum WGS-84, en la zona 18S. La provincia de Napo, es una de las provincias de la Región Centro Norte, situada principalmente en la región amazónica ecuatoriana, incluyendo parte de las laderas de los Andes.

El estudio combinó metodología cualitativa y cuantitativa, con el objetivo de reunir el máximo de información que permitiera explicar la situación del turismo de aventura en el Tena. Las fuentes de información que se han trabajado nos han permitido tener un amplio marco referencial sobre el turismo de aventura en el Tena, según el Plan Estratégico de Desarrollo para el Turismo Sostenible del Ecuador al 2020 (PLANDETUR 2020), y el Plan Integral de Marketing Turístico de Ecuador (PIMTE 2014). Turísticamente, la provincia de Napo, Amazonía Norte, se han consolidado como producto "B", es decir, cuenta con gran afluencia de turistas nacionales y extranjeros. De acuerdo con las estadísticas llevadas por la Unidad de Turismo del Cantón Tena, la afluencia turística a la provincia de Napo fue de 83,659 turistas durante el año 2010, último levantamiento de información.

Para el estudio, en 2014 se realizaron encuestas a turistas nacionales y extranjeros, consi- 
derando como universo 83,659 turistas nacionales y extranjeros, se trataron los datos a nivel estadístico, determinando una muestra de 100 encuestas, con un error de la muestra deseado del $10 \%$ y un nivel de confianza del 95\%. Las preguntas de las encuestas fueron cerradas, y las variables resultantes fueron edad, género, procedencia, estado de los atractivos, estado de las vías, seguridad, alimentos y bebidas, alojamiento, distribución del gasto y satisfacción general del turista. Los resultados fueron procesados a través de métodos descriptivos, base de la investigación del presente artículo.

\section{Resultados y discusión}

\subsection{Deportes de aventura en el Tena}

El Ecuador es un país privilegiado y propicio para realizar turismo de aventura, actividades que ofrecen al turista la posibilidad de experimentar las emociones y adrenalina propia de un deporte extremo, tales como: rafting, kayak, trekking, ciclismo de montaña, y ofrece una gran variedad de viajes de cross country, descenso, puenting, tours y excursiones MTB. A continuación se describen algunas de estas actividades.

En la ciudad del Tena, los deportes de aventura más conocidos son rafting y kayak, pues los ríos que nacen en las cumbres nevadas de los volcanes Antisana y Cotopaxi se desplazan vertiginosamente a los dos lados de los Andes, hacia el Pacífico y hacia la inmensa llanura amazónica. Adicionalmente, cada bote de rafting es acompañado de un kayakero de seguridad, el cual brinda apoyo logístico durante el trayecto en los ríos de aguas rápidas, haciendo uso de intercomunicadores para mantener contacto con el equipo en tierra, permitiendo brindar una experiencia optima y satisfactoria en los ríos del cantón Tena. Los ríos donde se lleva a cabo esta actividad deportiva de aventura son el rio Jatun Yacu, catalogado como uno de los mejores ríos para esta actividad, el rio Anzu, de un grado de dificultad media, y el rio Misahuallí, de grado de dificultad avanzado.

El puenting es una actividad que ofrece al turista la posibilidad de experimentar las emociones y adrenalina propia de un deporte extremo. El puenting es un columpio gigante, que consiste en realizar un salto tipo pendular desde un puente amarrado con cuerdas dinámicas, y equipos homologados y estandarizados que cumplen todas las regulaciones internacionales.
Se cuenta con unos pocos metros de caída libre hasta que las cuerdas se tensionan. La emoción que provoca el puenting es casi indescriptible. Como en un columpio para niños, nunca hay “templón”, siguiendo el recorrido del péndulo y sin rebote.

El canyoning, o descenso por cascadas, es otro tipo de deporte de aventura. Este deporte consiste en el descenso de cascadas con cuerdas, actividad que permite sentir la caída del agua en el rostro y el cuerpo, con la agradable sensación que produce sentir el agua fluir. No necesita experiencia ni condición física para disfrutar al máximo de esta aventura.

El ciclismo de montaña ofrece una gran variedad de viajes de cross country, descenso, tours y excursiones MTB para ciclistas. Existe la posibilidad de realizar estas actividades en uno de los ecosistemas mega diversos del planeta, como es la Amazonia ecuatoriana, atravesando el bosque nublado de la selva amazónica. El visitante tiene la posibilidad de conocer varias parroquias rurales como Talag, Pano y Muyuna. La ruta de ciclismo más requerida y ofertada va desde la parroquia Pano hacia la parroquia Muyuna, por una vía de segundo orden que atraviesa un paisaje de variados ríos, tiene una distancia de 32 $\mathrm{km}$, y un grado de dificultad medio.

La observación de aves o birdwhatching es una de las actividades de mayor acogida dentro de la demanda de los visitantes en Tena, debido a la gran variedad de especies de aves existentes, como loros y pericos, los cuales pueden ser apreciados en los denominados "saladeros de aves". Estos son sitios donde estas aves llegan a consumir barro por sus propiedades digestivas, que hacen efecto en su organismo, ayudando a las aves a eliminar toxinas que son ingeridas en su dieta de varios frutos silvestres. En el cantón existen infinidad de estos sitios, de los cuales los más conocidos se encuentran localizados en la parroquia Talag (saladero de Icho Urco). Para aprovechar y participar de esta experiencia es necesario estar provisto de elementos simples como unos buenos binoculares y una cámara de alta resolución, además de predisponerse a efectuar una caminata de dificultad media por la selva amazónica. Es una de las actividades de mayor demanda puesto que los turistas nacionales, y sobre todo extranjeros, pagan altos precios por lograr vivir esta experiencia.

El trekking es muy común en las zonas que poseen un entorno abundante de flora y fauna, así como paisajes y bellezas escénicas únicas y hermosas cas- 
cadas. Caminar a lo largo de senderos ecológicos crea una sensación de contacto con la naturaleza mucho más íntima entre el visitante y el medio natural. Este tipo de senderos se encuentran ubicados en varias de las áreas naturales existentes en el cantón Tena y sus parroquias, además de existir senderos ecológicos para la visita de atractivos en zonas privadas. La observación de especies de flora como orquídeas, bromelías y heliconias, son una actividad que se desarrolla en la mayoría de las ofertas turísticas, incluido el conocimiento de plantas ancestrales y medicinales de la zona.

El tubbing es una actividad que va tomando auge en la localidad, es el descenso en balsas neumáticas por las cuencas de ríos de niveles intermedios de dificultad (clase 1 y 2). En la zona se lo realiza en la cuenca alta del río Tena, el Pano y el Napo.

\subsection{Turistas en el Tena}

El bajo nivel de desarrollo promocional por parte de las agencias de viajes y otros actores del sector turístico ha traído como consecuencia la baja afluencia de turistas al destino Tena.

A través de la encuesta se llegó a determinar que el $60 \%$ de turistas son hombres y el $40 \%$ son mujeres, que llegan a la provincia a realizar caminatas y excursiones en la selva, entre las edades de 25 y 41 años, motivados por las actividades que se ofertan (Tabla 2). El lugar de origen de estos turistas son ecuatorianos, con un $83 \%$, seguido de turismo internacional, con el $17 \%$, por lo que se demostró que la provincia del Tena necesita mayor promoción internacional.

Tabla 2. Procedencia de los turistas encuestados. Diciembre de 2014.

\begin{tabular}{cccccc}
\hline Informantes & Resultados & Informantes & Resultados & Informantes & Resultados \\
\hline Ecuador & 83 & Alemania & 2 & Ucrania & 1 \\
EE.UU & 5 & Austria & 2 & España & 1 \\
Colombia & 3 & Francia & 2 & Perú & 2 \\
\hline
\end{tabular}

Los encuestados calificaron al servicio turístico como excelente en un $40 \%$, y bueno en un 55\% (Tabla 3). El estado de las vías es excelente en un 31\% y bueno en un $45 \%$ en vías de primer orden. La seguridad del visitante es buena en un 54\%. Los alimentos y bebidas son buenos para el $51 \%$ de los encuestados, y el alojamiento es considerado excelente en un $26 \%$, en relación con la comodidad que el encuestado percibe en hoteles y lodges, y un $21 \%$ no pernocta en la localidad. El nivel de satisfacción de los turistas es del $68 \%$, y el 100\% de ellos recomendarían visitar la provincia del Tena a amigos y familiares.

Tabla 3. Satisfacción de los encuestados con los servicios recibidos. Diciembre de 2014.

\begin{tabular}{|c|c|c|c|c|c|c|}
\hline Categorias & Estado de atractivo & Vías & Seguridad & $\begin{array}{c}\text { Alimentos y } \\
\text { bebidas }\end{array}$ & Alojamiento & $\begin{array}{c}\text { Satisfacción } \\
\text { servicio }\end{array}$ \\
\hline Excelente & $40 \%$ & $31 \%$ & $32 \%$ & $27 \%$ & $23 \%$ & $30 \%$ \\
\hline Bueno & $55 \%$ & $45 \%$ & $54 \%$ & $51 \%$ & $46 \%$ & $63 \%$ \\
\hline Regular & $3 \%$ & $20 \%$ & $23 \%$ & $15 \%$ & $10 \%$ & $5 \%$ \\
\hline Malo & $0 \%$ & $4 \%$ & $0 \%$ & $1 \%$ & $0 \%$ & $0 \%$ \\
\hline Muy Malo & $0 \%$ & $0 \%$ & $0 \%$ & $0 \%$ & $0 \%$ & $0 \%$ \\
\hline No ha visitado & $2 \%$ & $0 \%$ & $1 \%$ & $63 \%$ & $21 \%$ & $4 \%$ \\
\hline TOTAL & $100 \%$ & $100 \%$ & $100 \%$ & $100 \%$ & $100 \%$ & $100 \%$ \\
\hline
\end{tabular}

La distribución del gasto principalmente se la divide en alojamiento, alimentos y bebidas y deportes de aventura, con un 56\% (Tabla 4). El $25 \%$ de las personas encuestadas manifiestan que su mayor inversión lo realizan en alimentos y bebidas (A\&B), aunque también invierten para realizar actividades recreacionales como son los deportes de aventura y alojamiento. 
Tabla 4. Gasto de los encuestados por actividad o servicio. Diciembre de 2014.

Del $100 \%$ de su gasto total, indique los porcentajes gastados en:

\begin{tabular}{|c|c|c|}
\hline Categoria & Frecuencias & Porcentajes \\
\hline Alojamiento & 15 & $15 \%$ \\
\hline $\mathrm{A} \& \mathrm{~B}$ & 25 & $25 \%$ \\
\hline Guías & 9 & $9 \%$ \\
\hline Servicios Recreacionales & 9 & $9 \%$ \\
\hline Deportes de Aventura & 16 & $16 \%$ \\
\hline Artesanías & 8 & $8 \%$ \\
\hline Transporte & 12 & $12 \%$ \\
\hline Alquiler de Equipos & 0 & $0 \%$ \\
\hline Entradas & 6 & $6 \%$ \\
\hline TOTAL & 100 & $100 \%$ \\
\hline
\end{tabular}

Fuente: elaboración propia

A partir de los resultados de la investigación, se evidencia que se requiere incrementar el trabajo promocional del cantón Tena, comprometiendo en este trabajo a los municipios locales y agencias de viajes a través de un plan promocional internacional, tanto para mercados europeos como americanos. Dicho plan debería enfocarse en mostrar las bondades naturales que posee la localidad con el objetivo de incrementar la afluencia de visitantes, que conlleve a generar fuentes de empleo alternativas a los pobladores. Para lograr un manejo adecuado de los recursos naturales y culturales es importante primero una adecuada organización del territorio. Además, se requiere el incremento de fuentes de información primaria acerca de la demanda que registra el cantón, ya que esta información no la posee el Ministerio de Turismo o la Cámara de turismo del sector, por lo que los datos y fuentes de información son muy reducidos, haciendo muy difícil el levantamiento de información para la realización del diagnóstico del territorio.

\section{Conclusiones y recomendaciones}

La presente investigación busca dar a conocer la realidad de las actividades turísticas en la localidad, y determinar las problemáticas existentes, que obstaculizan en la actualidad el desarrollo del turismo de aventura. La principal problemática que existe es la falta de fuentes de información primaria acerca de la demanda que registra el cantón, limitando la obtención de información útil y de aplicación inmediata.

Se recomienda al GAD-TENA tomar medidas ambientales respecto del manejo de las cuencas hidrográficas del cantón, que se encuentran en proceso de contaminación por la falta de alcantarillado sanitario, y por el manejo inadecuado de los residuos y desechos sólidos, puesto que los ríos y acuíferos son la base del desarrollo de estas actividades de aventura.

Se recomienda a las autoridades del MAE-TENA realizar controles sobre el incremento de actividades extractivas de oro en los ríos Latunyacu y Anzu, principales ríos aprovechados para actividades de rafting, tubbing y kayaking. Así como también, la implementación de planes de bio-remediación de los ríos Pano y Tena, principales ríos visitados por los turistas locales y foráneos en las cercanías de la ciudad.

Se recomienda trabajar en la vinculación institucional de entidades públicas y privadas, a fin de fortalecer la planificación de las Juntas Parroquiales del Cantón Tena, para trabajar de manera conjunta en relación con las actividades de turismo de aventura.

Se plantea implementar una campaña de difusión del Cantón en medios de comunicación local y nacional para fomentar la visita a este del lugar. 


\section{Referencias}

Asociación de Municipalidades del Ecuador - AME. (2014). Historia del Cantón Tena. Obtenido de Cantón Tena: http://www.ame.gob.ec/ame/index.php/ ley-de-transparencia/57-mapa-cantones-del-ecuador/ mapa-napo/200-canton-tena

Martínez, A. S. \& Fernández, R. (2011). Estudio comparativo de empresas de turismo de aventura de la provincia de Valencia. Gran Tour: Revista de Investigaciones Turísticas, 3, 51-68.

Mediano, L, (2004). La Gestión de Marketing en el Turismo Rural. $1^{\text {a }}$ Edicion. Editorial Pearson Educación

MINCETUR. (2015). Conceptos fundamentales de turismo. Obtenido de: http://www.mincetur.gob.pe/ TURISMO/Producto_turistico/Fit/fit/Guias/Amazonas.pdf

MINTUR (2014). Operación Turística de Aventura en Ecuador cuenta con reglamento. Obtenido de: https://www.turismo.gob.ec/operacion-turistica-de-aventura-en-ecuador-cuenta-con-reglamento/

Mora, M. Ruiz, C. Palacios, Y. \& Yépez, R. (2014). Plan de Desarrollo y Ordenamiento Territorial Tena. GAD Tena.
Olivera, J. (1995). Las actividades físicas de aventura en la naturaleza: análisis sociocultural, Revista Apuntes, 41, 5-8.

Organización Mundial del Turismo - OMT. (1998). Introducción al Turismo. Publicación de la OMT en Educación Turistica. $1^{\text {a }}$ Edición.

Ron, K. (2009). Diseño de un plan de marketing turístico para el cantón Tena, provincia de Napo. Tesis de grado previa a la obtención del título de Ingeniero de empresas turísticas y áreas naturales. Universidad Tecnológica Equinoccial. Facultad de Turismo y Preservación Ambiental, Hotelería y Gastronomía, Carrera de Turismo y Preservación Ambiental.

Sung, H. Morrison, A. O'Leary, J. (1996) Definición de turismo de aventura. Disponible en URL: http:// www.ilustrados.com/tema/3834/turismoaventura. htm [consulta 11 de febrero del 2015] 